\title{
Multiple lasing Brillouin Fiber Laser with the implementation of reflective Fiber Bragg Grating in a ring cavity configuration
}

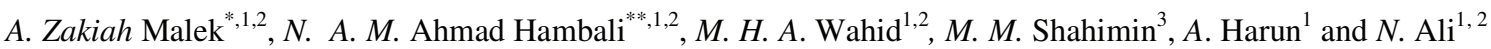 \\ ${ }^{1}$ School of Microelectronics Engineering, Universiti Malaysia Perlis (UniMAP), 02600 Arau, Perlis, Malaysia \\ ${ }^{2}$ Semiconductor Photonics \& Integrated Lightwave Systems (SPILS), School of Microelectronic Engineering, Universiti Malaysia \\ Perlis, Pauh Putra Main Campus, 02600 Arau, Perlis, Malaysia \\ ${ }^{3}$ Department of Electrical and Electronic Engineering, Faculty of Engineering, National Defence University of Malaysia (UPNM), \\ Kem Sungai Besi, 57000 Kuala Lumpur.
}

\begin{abstract}
A multi-wavelength Brillouin fiber laser with the implementation of reflective fiber Bragg grating is developed. In this ring cavity architecture, the fiber laser system is performed by the stimulated Brillouin scattering effect and four-wave mixing process. An improvement in the number Brillouin Stokes signal and average of the optical signal to noise ratio were generated through the reflection process of fiber Bragg grating. Due to the optimization of output coupling ratio from $10 \%$ to $60 \%$, the highest Brillouin Stokes signal of 17 and average optical signal to noise ratio of $14.7 \mathrm{~dB}$ were recorded at $60 \%$ of the output coupling ratio. The amplified Brillouin pump power of $16.5 \mathrm{dBm}$ at $1550 \mathrm{~nm}$ of Brillouin pump wavelength was injected into the laser system. A constant line spacing of $0.08 \mathrm{~nm}$ at each of the Brillouin Stokes signals was also recorded.
\end{abstract}

\section{Introduction}

Multi-wavelength fiber lasers can be defined as laser sources that emitting light more than one signal or multiple Brillouin Stokes (BS) signals from a main single wavelength light source [1]. The multiwavelength generation is commonly capable producing an accurate narrow linewidth that made them offered several high potentials in an optical communication system especially in dense wavelength division multiplexing (DWDM) application [2]. As mentioned in a previous paper [2], the operation of DWDM system was manipulated from the extreme growth of multiple signals in order to attain an efficient operation and consequently to make the DWDM system as one of the attractive technology among the researchers. Other notable technologies that applied the multi-wavelength lasers are mostly found in microwave signal processing [3], optical sensors, optical spectroscopy, current monitors, short optical pulses [4] and high capacity optical fiber communication networks [3].

Recently, the requirement for multiwavelength Brillouin fiber laser (MWBFL) is highly desirable for the optical communication system due to its ability to counter the limitations in the multiwavelength BrillouinErbium fiber laser (MWBEFL) and multiwavelength Brillouin-Raman fiber laser (MWBRFL). In such a situation, the major drawback of MWBEFL is related to the intrinsic generation of self-lasing cavity modes at the peak of the Erbium-doped fiber (EDF) gain [5] meanwhile a spectrum broadening effect in the MWBRFL cavity is another issue need to be considered [6]. Consequently, these limitations have a major impact on the formation of multi-wavelength generation. In an effort to increase the performance of the laser system, the hybrid MWBFL can counter these problems by allowing a higher coherent light source with narrow downshifted signal through stimulated Brillouin scattering (SBS) effect [7] and thus offer a solution to produce a larger number BS signal.

The direction of MWBFL studied was demonstrated in both linear and ring cavity configurations. Various ring cavity configurations of MWBFL system have been previously reported in [1, 8-9]. In this reported work [1], the ring cavity was successfully produced up to $8 \mathrm{BS}$ signal with $0.08 \mathrm{~nm}$ line spacing by a combination of forward and backward output signals. Meanwhile, 0.16 $\mathrm{nm}$ of line spacing in between two consecutive odd or even BS signals was attained when the forward and backward direction were discriminated from the laser system. Another invention also proposed by M. R. Nurdik and his team [8]. This ring cavity BFL was mainly constructed by using the same type and length (25 km of SMF-28) of the Brillouin gain medium as previously reported in [1]. The performance of laser system was achieved with 10 BS signals and 7 antiStokes signals at a line spacing of $0.088 \mathrm{~nm}$ by launching $11.29 \mathrm{dBm} \mathrm{BP}$ power at $1510 \mathrm{~nm}$ of $\mathrm{BP}$ wavelength. Besides, at a BP wavelength of $1550 \mathrm{~nm}$, the limitation of multi-wavelength generation occurred

\footnotetext{
Corresponding author: *amirazakiahmalek@gmail.com and **azuramalini@unimap.edu.my
} 
when only 5 BS signals were extracted at $50 \%$ output coupling ratio.

A linear cavity of BFL with multiple wavelength outputs was also reported in [10]. The optimization of output coupling ratios was varied from $70 \%$ to $99 \%$ in order to control the amount of light propagation. The effect of 95:5 coupling ratio produced up to $11 \mathrm{BS}$ signals and anti-Stokes signals simultaneously with a line spacing of $0.08 \mathrm{~nm}$. A maximum BP power of 11.7 $\mathrm{dBm}$ with $1550 \mathrm{~nm} \mathrm{BP}$ wavelength was chosen to inject into the laser cavity. In this paper, as compared to our previous configurations [1, 8-10], we successfully reported the increment number of BS signals with an acceptable average optical signal to noise ratio (OSNR) value. The employment of a fiber Bragg grating's (FBG) reflectivity offers a direct impact to produce better lasing performance in a ring cavity. The reflectivity and selective wavelengths of FBG enhance the SBS effect in the Brillouin gain medium to generate a higher nonlinear effect with $17 \mathrm{BS}$ signals and $14.7 \mathrm{~dB}$ of average OSNR value. A constant line spacing of $0.08 \mathrm{~nm}$ was also observed.

\section{Experimental Setup}

The architecture of ring cavity MWBFL with the implementation of a reflective FBG is illustrated in Fig. 1. It consists of an external tunable laser source (TLS), a unit of erbium-doped fiber amplifier (EDFA), $8 \mathrm{~km}$ of single mode fiber (SMF) that acts as a Brillouin gain medium, 3-port of optical circulator, $2 \times 2$ optical coupler and $3 \mathrm{~dB}$ bandwidth of $5 \mathrm{~nm}$ FBG with $70 \%$ reflectivity is used as another fiber loop mirror. Based on the FBG's properties, it only reflected the $5 \mathrm{~nm}$ wavelengths back at the centre wavelength of $1550 \mathrm{~nm}$, while others wavelengths would be filtered and eliminated from the laser system.

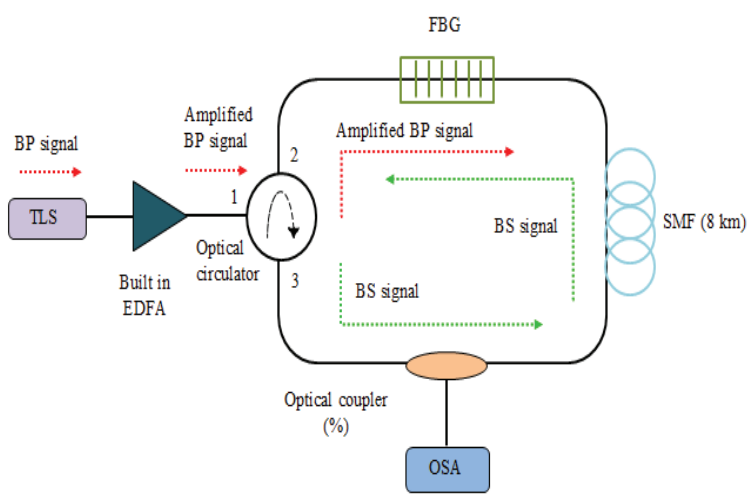

Fig. 1. The proposed ring cavity MWBFL system with the implementation of a reflective FBG.

In this experiment, the main nonlinear phenomenon called as SBS effect initiated in the SMF once the intensity of Brillouin pump (BP) signal is sufficient enough to excite the atoms. Without sufficient high BP power from the external TLS, the BP signals would be absorbed by the EDFA instead of being amplified by it.
After being amplified, the high BP signals oscillated into the port-2 via port- 1 of the optical circulator. From port2 of the optical circulator, the amplified BP signals propagated directly into the $\mathrm{FBG}$ in order to remove the unwanted wavelengths according to the FBG's properties. About $30 \%$ of amplified BP signals continued to transmit into the SMF while another $70 \%$ of amplified BP signals reflected in the opposite direction.

In the SMF, the SBS effect only generated when the Brillouin threshold condition is achieved. The first downshifted BS signal would propagate in the opposite direction of amplified BP signals and enter the FBG for the reflection process. Later, the first BS signal oscillated to the optical circulator and circulated back into the FBG and SMF to complete clockwise and anti-clockwise light propagation. This propagation continues until the power of subsequent BS signals is high enough to overcome the Brillouin threshold power. In other words, the first BS signal acts as a BP power to generate the second BS signal, and this propagation continues as long as the Brillouin gain is equal or greater than the cavity loss. This MWBFL system has a line spacing of approximately $0.08 \mathrm{~nm}$ which depends on the type of optical fiber (SMF). The output BS signal is extracted from the optical coupler form $10 \%$ to $60 \%$ and is monitored by using an optical spectrum analyzer (OSA) with $0.02 \mathrm{~nm}$ resolution bandwidth.

\section{Results and Discussion}

The corresponding optical spectrum of FBG's reflection profile was investigated as shown in Fig. 2. The $3 \mathrm{~dB}$ bandwidth of $5 \mathrm{~nm}$ is utilized in order to enhance the signal quality by having $70 \%$ of reflectivity. The improvement of light intensity generated within $1547 \mathrm{~nm}$ to $1553 \mathrm{~nm}$. There is a stop band of wavelengths in which filtered certain wavelengths from propagated into the Bragg grating. Thus, only desired wavelengths that fulfilled the Bragg's condition are strongly back-reflected.

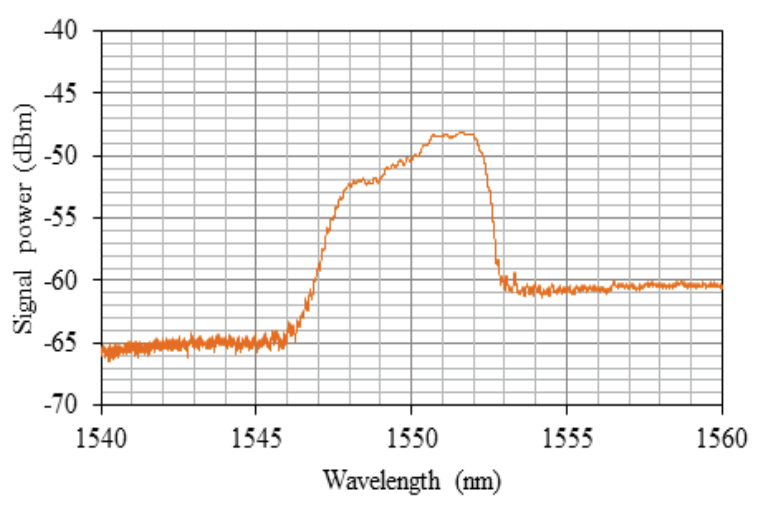

Fig. 2. The optical spectrum of FBG's reflection profile.

As the input light of $15.5 \mathrm{dBm}$ with a broad spectrum was propagated along the core of the optical fiber, there was only $1547 \mathrm{~nm}$ to $1553 \mathrm{~nm}$ that back-reflected light at maximum reflectance at Bragg wavelength, $\lambda_{B}$. 
Meanwhile, the transmitted light would eliminate from the spectrum due to Bragg's condition. It should be noted that the light propagation in the FBG was not only occurred in the back-reflected direction but also occurred in the forward direction.

Next, the effect of amplified BP power on the generation of multi-wavelength was investigated as depicted in Fig. 3. In this investigation, the amplified BP power is considered as the input signal after being amplified by the EDFA. The amplified BP power was varied from $12.0 \mathrm{dBm}$ to $16.5 \mathrm{dBm}$ whereas the $\mathrm{BP}$ wavelength was fixed at $1550 \mathrm{~nm}$ and $60 \%$ of the output coupling ratio was used to extract the output signal. Referring to Fig. 3, the number of BS signals increases as the amplified BP power gradually increases. This condition can be explained as the EDFA provides high Brillouin gain for initiating the maximum SBS effect. Due to the increment of the amplified BP power, the SBS effect generates efficiently to produce the downshifted BS signal in the opposite direction and thus more multi-wavelength generation was observed.

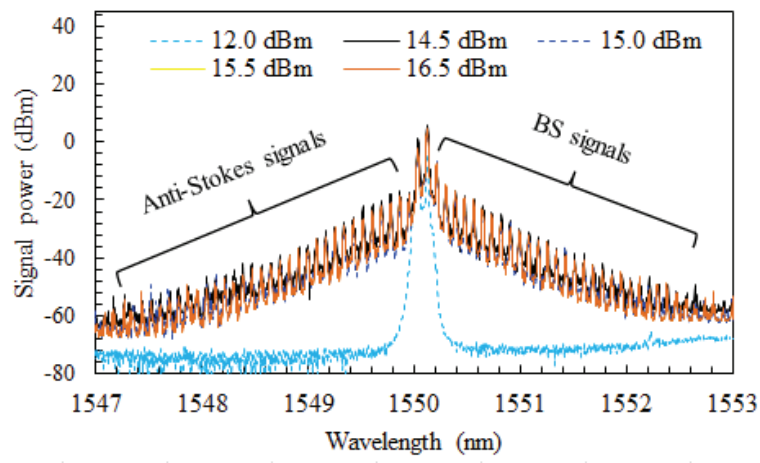

Fig. 3. Output spectra for different amplified BP powers at $1550 \mathrm{~nm}$ of BP wavelength by utilized $60 \%$ of the output coupling ratio.

At $12.0 \mathrm{dBm}$ of amplified BP power, the optical spectrum indicates only 1 BS signal because of low injected input power. The BS signal generated once the Brillouin threshold power is satisfied [11]. In this case, at $12.0 \mathrm{dBm}$ of amplified BP power, the laser system is considered as around Brillouin threshold condition. When the amplified BP power was increased further, it was found that the generation of multi-wavelength was started to generate with $8 \mathrm{BS}$ signals at $14.5 \mathrm{dBm}$. It was noticed that the amount of power at each of the BS signals were dropped due to low Brillouin gain. By increasing the amplified BP powers from $15.0 \mathrm{dBm}$ to $16.5 \mathrm{dBm}$, more multi-wavelength were generated. The most generated BS signals of 17 were found at maximum amplified BP power of $16.5 \mathrm{dBm}$. Besides that, it was also noticed that the anti-Stokes signals were generated at the left side of the optical spectra for 14.5 $\mathrm{dBm}$ to $16.5 \mathrm{dBm}$. The formation of anti-Stokes signal happened based on the interaction of multiple four-wave mixing (FWM) process. The unidirectional propagation in the laser system produced the anti-Stokes signal where the BS signal interacts with a pump beam to create other new signals (anti-Stokes signal). The number of antiStokes signals is lower compared to BS signals because the FWM process depends on the BS signals. The antiStokes signals increases as the BS signals increases.

The relationship of number BS signal with different amplified BP powers by utilizing $8 \mathrm{~km}$ of SMF is illustrated in Fig. 4. In this study, the BS signal experienced a flat trend before having a dramatic growth for all proposed output coupling ratios. At amplified BP powers of $12.0 \mathrm{dBm}$ to $13.5 \mathrm{dBm}$, only $1 \mathrm{BS}$ signal was recorded for all case output coupling ratios. At amplified $\mathrm{BP}$ power of $14.0 \mathrm{dBm}$ to $16.5 \mathrm{dBm}$, the number $\mathrm{BS}$ signals were dramatically increased. This observation has resulted because of the higher Brillouin gain property accumulated higher SBS effect. This effective Brillouin gain medium provides maximum light intensities to lase the MWBFL system. Thus, this oscillated light resulted in the increment of the generated BS signals. The highest BS signal of 17 was recorded at $16.5 \mathrm{dBm}$ of amplified BP power. In terms of output coupling ratio factor, the $\mathrm{BS}$ signals improved as the coupling ratios were increased. This also corresponds to the higher output coupling ratio influenced the increment of cavity loss. Therefore, the oscillating energy experienced inside the ring cavity becomes lesser. As a result, the lowest number BS signal of 4 was found at $10 \%$ output coupling ratio with $16.5 \mathrm{dBm}$ of amplified BP power. Meanwhile, the highest number BS signal of 17 was attained at $60 \%$ optimum coupling ratio by launching $16.5 \mathrm{dBm}$ of amplified BP power.

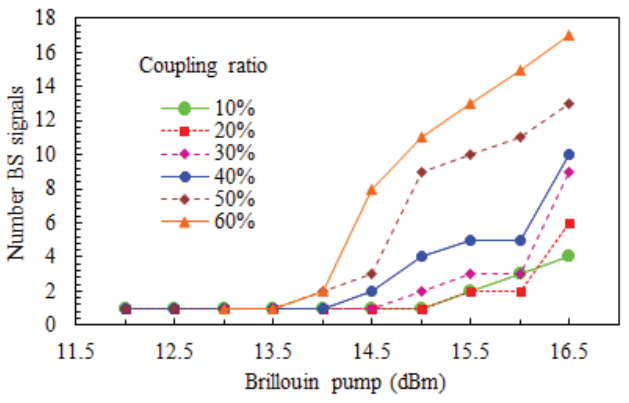

Fig. 4. Number BS signals as a function of amplified BP powers at $1550 \mathrm{~nm}$ of BP wavelength.

Fig. 5 depicts the average of OSNR with different amplified BP powers. Due to the effect of additional FBG's reflection, the average of OSNR gradually increases as the increment of amplified BP powers and output coupling ratios. The sufficient amount of amplified BP power that shared at each of the BS signals was the main factor that affected this abrupt change. At high amplified BP power, the laser system provided a high Brillouin gain which results in increasing of the OSNR. Therefore, high amplified BP power was required to supply the input power to reduce the noise level of the MWBFL system in which led to increases of OSNR. Moreover, the higher average OSNR is also owing to the implementation of additional FBG in which enhances the signal quality by having a reflection process. In this experiment, the average of OSNR was 
measured by comparing the peak power at each of the BS signals to the highest noise floor level of the signal [12]. The deduction of both signals is defined as the OSNR. Since the MWBFL system produced a multiwavelength generation, the OSNR for each of the BS signals was calculated and then divided into the total number BS signals in order to calculate the average OSNR. Overall, the lowest average of OSNR was obtained around $11.1 \mathrm{~dB}$ for $12.0 \mathrm{dBm}$ of amplified BP power at $10 \%$ of the output coupling ratio. Meanwhile, a higher average of OSNR about $14.7 \mathrm{~dB}$ was achieved for $16.5 \mathrm{dBm}$ of amplified BP power and $60 \%$ of the output coupling ratio.

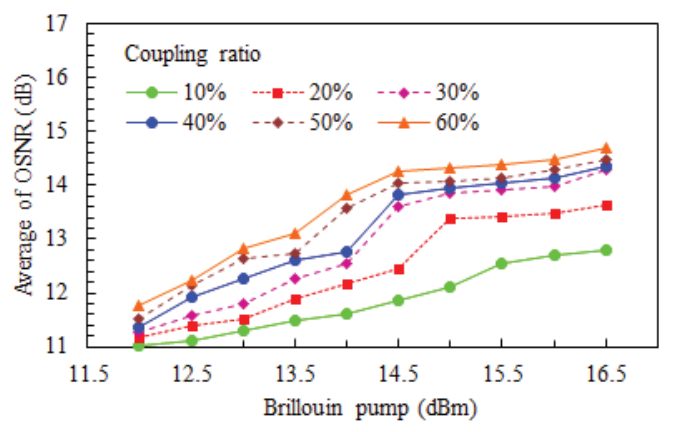

Fig. 5. Average of OSNR with different amplified BP powers for all cases output coupling ratios.

\section{Summary}

We have successfully demonstrated a ring cavity MWBFL system with the implementation of a reflective FBG that can be used at $1550 \mathrm{~nm}$ in the optical communication window. At amplified BP power of 16.5 $\mathrm{dBm}$ with a corresponding $60 \%$ of the output coupling ratio, $17 \mathrm{BS}$ signals and $14.7 \mathrm{~dB}$ average of OSNR were generated by the combination process of multiple SBS and FWM effect. The output BS signals were constantly separated by $0.08 \mathrm{~nm}$. The implementation of FBG inside the ring cavity can effectively be used in the laser system especially in the Brillouin fiber laser to increase the number BS signals. Moreover, due to the reflection process of FBG, an acceptable average of OSNR was recorded which could be used for several potential applications in the optical communication. For future work, a high reflection of FBG can be utilized to improve the average OSNR in order to meet the requirements of high capacity optical communication networks.

This work was fully supported by the Ministry of Higher Education, Malaysia under research grant \# FRGS/900300532\#. The authors would like to thank the School of Microelectronics Engineering, Universiti Malaysia Perlis (UniMAP) especially SPILS for their support in this work.

\section{References}

1. M. R. Shirazi, M. Biglary, S. W. Harun, K. Thambiratnam and H. Ahmad, J. of Optic A: Pure and Applied Optics 10, 5 (2008).

2. A. Aloisio, F. Cevenini and V. Izzo, IEEE Trans. Nucl. Sci. 51, 526-531 (2004).

3. Y. Shen, X. Zhang and P. A. Robert, J. Lightw. Tech., 23, 1860-1865 (2005).

4. Y. Shen, X. Zhang and K. Chen, J. of Lightw. Techn. 23, 1860 (2005).

5. S. J. Tan, S. W. Harun, N. M. Ali, A. Ismail and H. Ahmad, IEEE J. of Quantum Electro., 49, 595-598 (2013).

6. M. Ali. Toor, N. A. M. Ahmad. Hambali, A. Mansoor, M. Ajiya and Z. Yusoff, J. of Optics 17, 025502 (2015).

7. M. P. Fok and C. Shu, Optics Exp., 14, 2618-2624 (2006).

8. M. R. Nurdik, N. S. Abdul Rahim, and M. K. Abd Rahman, Brillouin fiber laser with 200-nm tuning range, IEEE 2nd Internatinal Conference on Photonics (ICP), p1-4 (2011).

9. B. A. Ahmad, A. W. Al-Alimi, A. F. Abbas, M. Mokhtar, S. W. Harun and M. A. Mahdi, IEEE Photonics J., 1087-1094 (2012).

10. M. R. Shirazi, N. S. Shahabuddin, S. N. Aziz, K. Thambiratnam, S. W. Harun and H. Ahmad, Laser Phys. Let., 5, 361-363 (2008).

11. B. A. Ahmad, Gh. Mamdoohi, A. F. Abas, M. Mokhtar and M. A. Mahdi, International Conference on Photonics (ICP), 195-198 (2012).

12. A. W, Al-Alimi, M. H. Yaacob, A. F. Abas, M. A. Mahdi, M. Mokhtar and M. H. Al-Mansoori, IEEE Photonics J., 5, (2013). 